\title{
The 6.9- $\AA$ Structure of GIpF: A Basis for Homology Modeling of the Glycerol Channel from Escherichia coli
}

\author{
Henning Stahlberg,*,1 Thomas Braun,*,1 Bert de Groot,† Ansgar Philippsen,‡ Mario J . Borgnia,§ \\ Peter Agre,§ Werner Kühlbrandt," and Andreas Engel*,2
}

\begin{abstract}
*M. E. Müller Institute for Microscopy, and ¥Department of Structural Biology, Biozentrum, University of Basel, Klingel bergstrasse 70, CH-4056 Base, Switzerland; †Max-Planck-Institute for Biophysical Chemistry, Am Fassberg 11, D-37077 Göttingen, Germany; $\S$ Department of Biological Chemistry, J ohns Hopkins University School of Medicine, Baltimore, Maryland 21205; and

"Max-Planck-I nstitute for Biophysics, Kennedyallee 70, D-60596 Frankfurt, Germany
\end{abstract}

Received October 7, 2000

The three-dimensional structure of GIpF, the glycerol facilitator of Escherichia coli, was determined by cryo-electron microscopy. The 6.9-A density map calculated from images of two-dimensional crystals shows the GIpF helices to be similar to those of AQP1, the erythrocyte water channel. While the helix arrangement of GIpF does not reflect the larger pore diameter as seen in the projection map, additional peripheral densities observed in GIpF are compatible with the 31 additional residues in loops $C$ and $E$, which accordingly do not interfere with the inner channel construction. Therefore, the atomic structure of AQP1 was used as a basis for homology modeling of the GIpF channel, which is predicted to be free of bends, wider, and more vertically oriented than the AQP1 channel. Furthermore, the residues facing the GIpF channel exhibit an amphiphilic nature, being hydrophobic on one side and hydrophilic on the other side. This property may partially explain the contradiction of glycerol diffusion but limited water permeation capacity. $\odot 2000$ Academic Press

Key Words: AQP; cryo-TE M; GIpF ; water channel.

\section{INTRODUCTION}

Osmoregulation in bacterial, plant, and animal cells requires the presence of membrane channels, specific for water and small non-ionic solutes. Expression of aquaporin-1 (AQP1, the water channel of human erythrocytes) in Xenopus oocytes demonstrated its water permeation capacity (Preston et al., 1992) and initiated many functional and structural studies of the rapidly growing aquaporin superfam-

\footnotetext{
${ }^{1}$ These authors contributed equally to this work.

2 To whom correspondence should be addressed. Fax: +41 61 267 2109. E-mail: Andreas.Engel@unibas.ch.
}

ily. Phylogenetic analyses revealed the existence of two clusters of subfamilies, the aquaporins (AQPs) and glycerol facilitators (GLPs) (Heymann and Engel, 1999; Park and Saier, 1996). Their sequences share an internal repeat, with each half comprising three transmembrane regions according to hydropathy profiling (Gorin et al ., 1984; Preston and Agre, 1991) and sequence conservation (Heymann and Engel, 2000). Two NPA motifs represent the prominent fingerprint of the highly conserved loops $B$ and $E$, predicted to fold back into the core of the protein from the extra- and intracellular side, forming a channel resembling an hourglass (J ung et al., 1994).

This model was confirmed by cryo-el ectron microscopy of two-dimensional (2D) crystals of AQP1, yielding three-dimensional (3D) maps at sufficient resolution to reveal the secondary structure of the channel (Cheng et al., 1997; Li et al., 1997; Walz et al., 1997). These maps show the tetrameric organization of monomers each comprising a right-handed bundle of six highly tilted transmembrane helices that surround loops $B$ and $E$, which fold back to meet in the center of the membrane. At $4.5-\AA$ resolution, parts of loops $B$ and $E$ were found to form two additional short helices within this central structure (Mitsuoka et al., 1999). The first atomic model of AQP1 reveals the site of water sel ectivity to be close to the center of the monomer (Murata et al., 2000), as predicted by the hourglass model. This eyelet is determined by four conserved hydrophobic residues in helix 1 (F24), helix 2 (I60), helix 5 (V176), and helix 4 (L 149), two hydrophobic residues in loop B (L 75) and loop E (I 191), and the two conserved Asp (N76 and N 192) of the NPA motifs.

The internal sequence repeat is reflected in a pseudo-twofold symmetry within the monomer, as well as in the nondirectional diffusion of solutes through the pore. This pseudo-twofold symmetry 


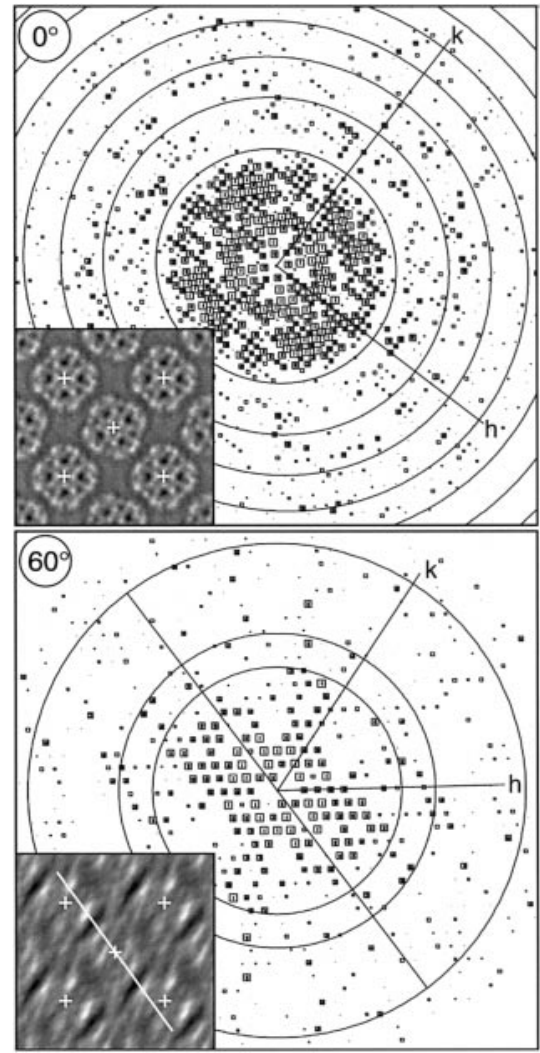

FIG. 1. Power spectra (IQ plots; Henderson et al., 1986) of the unbent images and calculated projection maps (insets) of an untilted sample (top) and a $60^{\circ}$ tilted sample (bottom). The reciprocal lattice axes are indicated ( $\mathrm{h}$ and $\mathrm{k}$ ). In the $0^{\circ} \mathrm{IQ}$ plot, the zero crossings of the contrast transfer function are marked by the concentric lines. In the $60^{\circ} \mathrm{IQ}$ plot, the circles indicate resolution limits of 7.5, 5.0, and $3.5 \AA$. The tilt axis is represented by a straight line in the IQ plot and in the projection map. The projection maps show four unit cells, which have dimensions $a=b=$ $104 \AA$. The crosses mark the centers of the tetramers.

also complicated the sidedness assignment. Atomic force microscopy before and after proteolytic cleavage of $\mathrm{N}$ - and/or C-termini allowed the sidedness of AqpZ, the bacterial water channel, and MIP, the channel of animal lens fiber cells, to be determined (Scheuring et al., 1999; Fotiadis et al., 2000). The sidedness of AQP1 was subsequently derived by comparing surface topography and projection maps of AqpZ with those of AQP1 (Scheuring et al., 2000) and by computationally fitting helical segments to the 4.5- $\AA$ map (de Groot et al., 2000), an assignment corroborated by the 3.8- $\AA$ structure (Murata et al., 2000).

The GLP subcluster is structurally less well characterized. Five key amino acids were found to distinguish between AQPs and GLPs (Froger et al., 1998). Two further amino acids (F 24 and L 149) were hypothesized to play a functional role (Heymann and Engel, 2000), which is now confirmed by the 3.8- $\AA$ structure. In the majority of GLPs (79\%), these residues are both $L$ eu, whereas they are mostly Phe and Leu in the AQPs. Another difference between AQPs and GLPs concerns long inserts in the extracellular loops C and E. Whereas all AQPs so far characterized are tetramers (Engel et al., 2000), it was speculated that the GLPs act as monomers (Bron et al., 1999; Lagree et al., 1998, 1999). However, studies of the solubilized glycerol channel GIpF from Escherichia coli (Braun et al., 2000) revealed a square-shaped particle similar in size and mass to those found for aquaporins (Hasler et al., 1998; Ringler et al., 1999; Walz et al., 1994). A tetrameric structure of GIpF also supports the model of direct interaction of the tetrameric glycerol kinase from $\mathrm{E}$. coli with GIpF (Voegele et al., 1993).

GIpF has rather broad substrate specificity (Maurel et al., 1994): Small uncharged compounds such as polyols and urea, but no ions such as protons or phosphorylated polyols, are able to diffuse through GlpF. How can this difference in permeability between GLPs and AQPs be explained? The 2D projection map of GIpF at 3.7- $\AA$ resolution revealed a larger central depression within the monomer than in AQP1 (Braun et al., 2000), compatible with a larger pore as required for the passage of polyols. To explore this question further we have determined the three-dimensional structure of GIpF at $6.9-\AA$ resolution. The striking structural similarity of GIpF with AQP1 and the sequence homology prompted us to build an atomic model of GIpF that appears to explain the specificity of the glycerol channel.

\section{MATERIALS AND METHODS}

Cryo-electron microscopy. GIpF was overexpressed in E. coli, purified on a Ni column, and 2D crystallized as described (Braun et al., 2000). For cryo-electron microscopy (Dubochet et al., 1988), grids were prepared by trehalose embedding (Hirai et al., 1999). All microscopic work was done within 10 days. Images were recorded at various tilt-angles up to $60^{\circ}$ with a J eol $3000 \mathrm{SFF}$ in spot scan mode, working at $300 \mathrm{kV}, 4.2 \mathrm{~K}$, and $70000 \times$ nominal magnification with an electron dose of approximately $17 \mathrm{e}^{-} / \AA^{2}$. The loss of resolution normal to the tilt-axis in images of tilted samples was significantly reduced by employing the spot-scan procedure. Negatives were examined with a laser diffractometer and digitized with a Zeiss Phodis scanner (Carl Zeiss, Oberkochen, Germany) at $1 \AA$ /pixel on the specimen level or with a Leafscan-45 scanner (Leaf Systems. Inc., Westborough, MA) at $1.4 \AA$ Åpixel.

I mage processing. I mage processing was performed using the MRC crystallography program suite (Henderson et al., 1986, 1990). I mages were corrected for lattice distortions, taking the F ourier-filtered images themselves as references. Phases and amplitudes were measured and corrected for the tilted contrast transfer function. I mages with two lattices of equal quality were processed for both crystal layers independently. Amplitudes and phases from processed images were merged imposing p4 symme- 

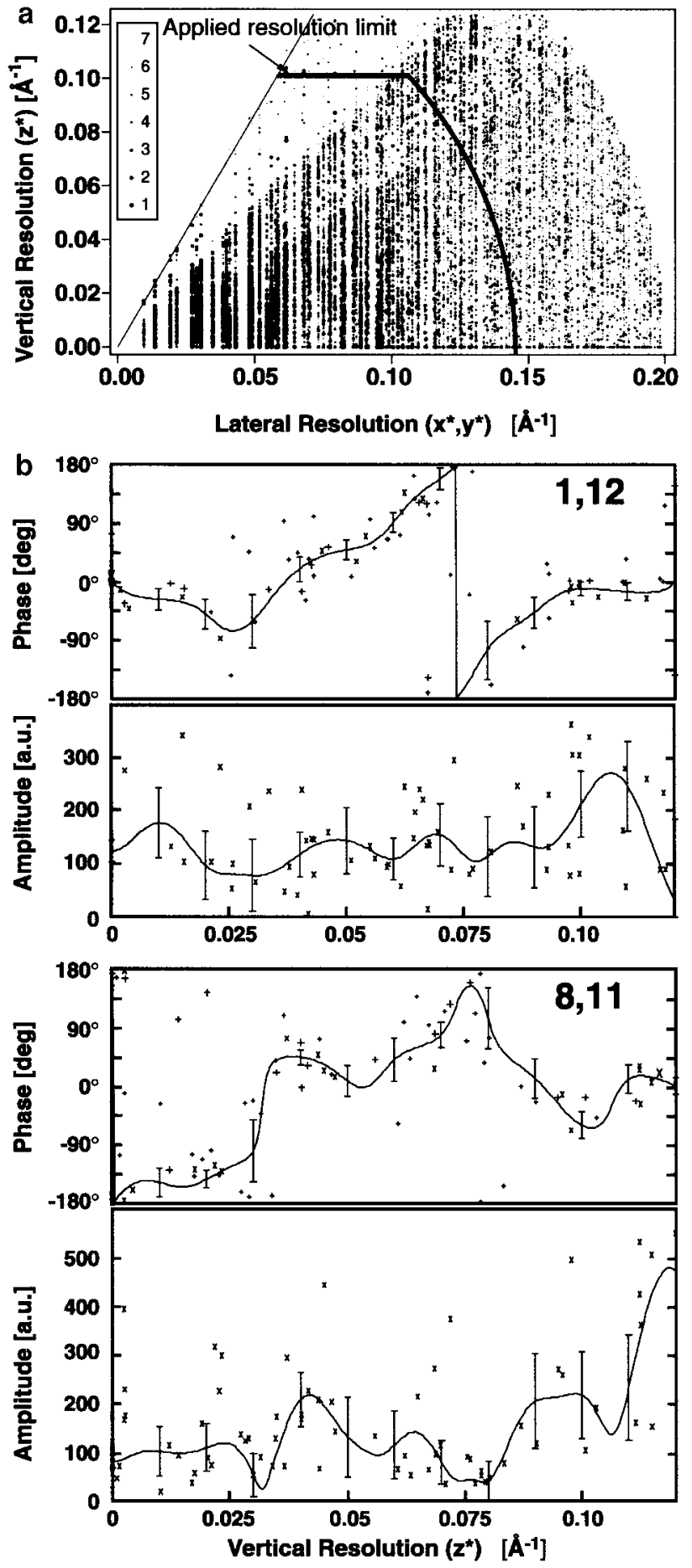

FIG. 2. (a) Azimuthal projection of the three-dimensional data in the asymmetric quadrant, showing the data sampling. The limit of the missing cone is marked by a thin line. The size of the data points indicates their IQ value (Henderson et al., 1986); a reference scale is given in the inset. The largest spots correspond to IQ = 1 and the smallest to IQ $=7$. Changes in sampling densities reflect the data collection at $30^{\circ}, 45^{\circ}$, and $60^{\circ}$. Despite the presence of data at higher resolution, the resolution limit was set to $(6.9 \AA)^{-1}$, in $z$-direction to $(10 \AA)^{-1}$, as indicated by the solid line (Table I). (b) Amplitudes and phases along the lattice line
TABLE I

Phase Residuals in Resolution Ranges

Crystallographic data

\begin{tabular}{ll}
\hline $\begin{array}{l}\text { Plane group symmetry } \\
\text { Unit cell }\end{array}$ & $\mathrm{p} 4$ \\
$\begin{array}{l}\text { Number of processed } \\
\text { images }\end{array}$ & 48 \\
$\begin{array}{l}\text { Number of processed } \\
\text { lattices }\end{array}$ & $73\left(0^{\circ}: 9 ; 30^{\circ}: 28 ; 45^{\circ}: 31 ; 65^{\circ}: 5\right)$ \\
$\begin{array}{l}\text { Number of merged phases } \\
\text { Maximum tilt angle }\end{array}$ & 16273 \\
Phase residual $^{\mathrm{a}}$ & $61^{\circ}$ \\
& ${\text { Overall: } 49^{\circ}}^{\left.\text {(IQ-weighted: } 25^{\circ}\right)}$
\end{tabular}

(200-6.9 А)

R-factor ${ }^{\mathrm{a}}(200-6.9 \AA)$

Resolution limit for merging

Completeness $^{b}$

Overall: 0.540 (IQ-weighted: 0.767)

In plane $(x, y): 6.9 \AA$

Normal to plane (z): $10 \AA$ $68.0 \%$

a Phase residuals, $\mathrm{R}$-factors, and number of merged phases were calculated with the program LATLINED.

${ }^{b}$ Only reflections within the resolution volume having a figure of merit over 0.5 were included; the missing cone comprises $13 \%$ of this volume.

try. After phase origin refinement, lattice lines for the amplitudes and phases normal to the membrane plane were fitted and sampled at $(100 \AA)^{-1}$ to create a 3D data set of structure factors. The 3D data set was used with the program MAKETRAN to create reference projections (Kunji et al., 2000). All images were then unbent with these synthetic references and merged again. A final 3D map was calculated using the CCP4 programs (Collaborative Computational Project, 1994).

Homology modeling. A model of the structure of GIpF was built based on the atomic structure of AQP1 (Murata et al., 2000) and an alignment of the two sequences (Heymann and Engel, 2000) using the WHATIF program (Vriend, 1990). Using the rotamer database of WHATIF, the side-chain conformations of W42 and $Y 138$ were refined manually to select the most probable rotamer state for both residues that did not block the channel. Rottrans (http://www.mpibpc.gwdg.de/abteilungen/071/bgroot/ maptools.html) was used to calculate the most favorable orientation of this model in the 3D el ectron crystallographic map of GIpF , and for this orientation, a projection in the membrane plane was calculated.

\section{RESULTS AND DISCUSSION}

\section{Data Collection and Processing}

Figure 1 shows IQ plots (Henderson et al., 1986) of an untilted and a $60^{\circ}$ tilted lattice recorded in a spot scan mode by a J eol 3000 SF F electron microscope. The corresponding projection maps were calculated after lattice unbending and contrast transfer func-

1,12 and 8,11 . The curves were calculated by a least-square algorithm from the experimental values of 73 images: 9 from untilted samples, 28 at $30^{\circ}$ tilt, 31 at $45^{\circ}$ tilt, and 5 at $60^{\circ}$ tilt. These curves were sampled at $(100 \AA)^{-1}$ to obtain a 3D data set of structure factors. The error bars are a measure of the data quality. 

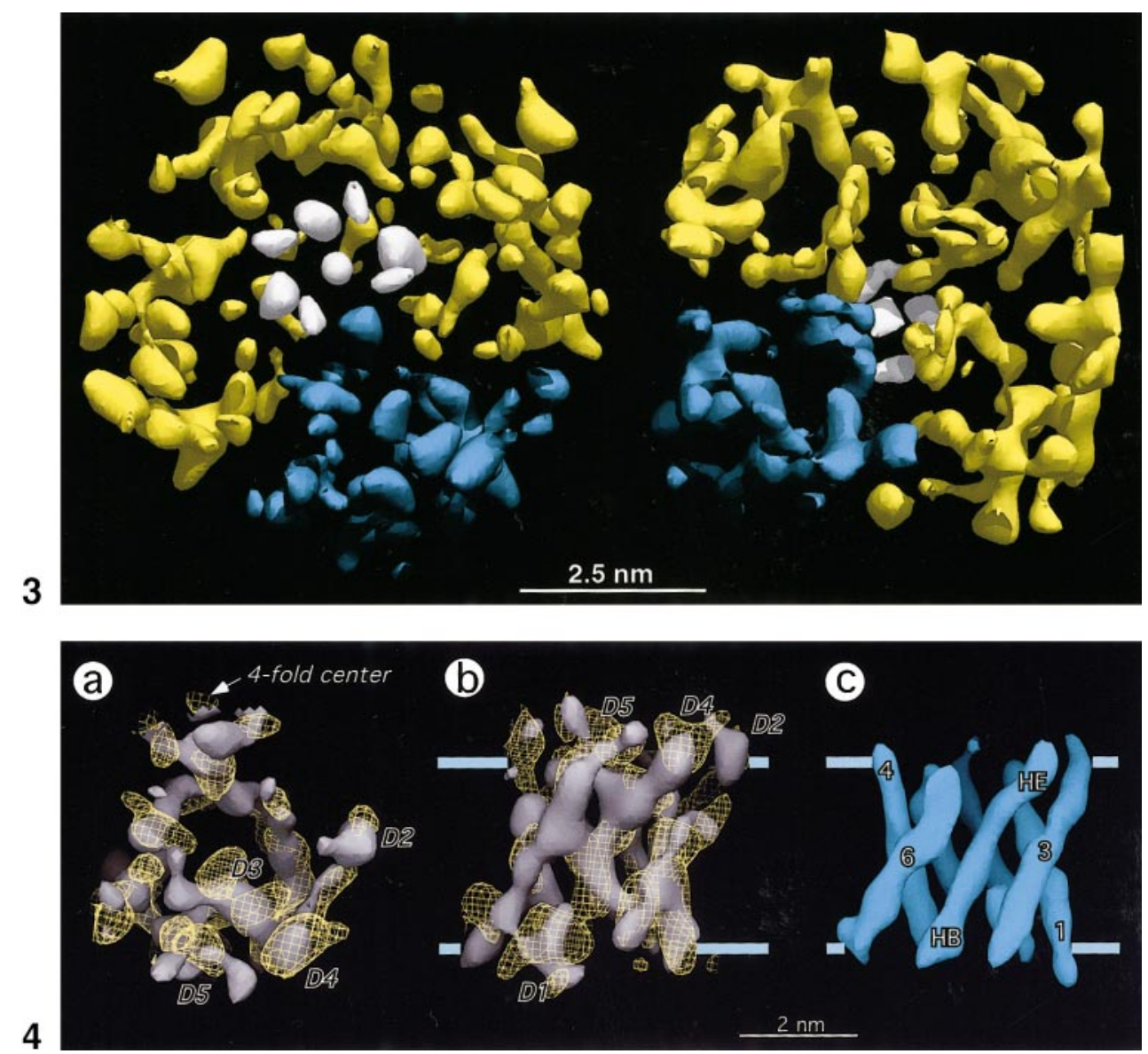

FIG. 3. The two oppositely oriented tetramers of one unit cell, seen at $20^{\circ}$ normal to the membrane plane. One monomer of each tetramer is highlighted in blue. The periplasmic density about the fourfold symmetry axis is shown in white. Due to the noncrystallographic symmetry between the two tetramers, data were merged imposing p4 symmetry. Rotating the left tetramer by $180^{\circ}$ around the horizontal axis and then by $30^{\circ}$ around its fourfold axis superposes it on the right tetramer. The in-plane distance between the tetramer centers is $73.5 \AA$.

FIG. 4. Comparison of independent $3 D$ density maps of GIpF with AQP1. The two independent GIpF monomers are represented as shaded and wire frame surfaces. (a) Top view from the periplasmic side. (b) Side view from outside the tetramer. (c) Equivalent side view of the AQP1 monomer rendered at 6.9- $\AA$ resolution. The membrane surfaces are indicated by light blue lines.

tion correction, using the MRC program package (Crowther et al., 1996). Since highly ordered GIpF lattices consisted always of two layers, both were processed, requiring determination of the sidedness.
This was unambiguous for the $0^{\circ}, 30^{\circ}$, and $45^{\circ} \mathrm{im}$ ages, but difficult for the $60^{\circ}$ images, of which only those five with clearly distinguishable sidedness were included in the merging process. After sided-

FIG. 5. Superposition of the averaged GIpF monomer (yellow shapes) with the AQP1 atomic model (colored $\alpha$-helices; Murata et al., 2000). Numbers indicate the helix segments of the primary sequence. The helix assignment was determined by cryo-electron microscopy (Murata et al., 2000) and also confirmed by sequence comparison (Heymann and Engel, 2000) as well as directionality pattern analysis (de Groot et al., 2000). Helical segments in loops B and E are indicated by HB and HE. Additionally identified densities are labeled D1 through D5; see text. (a,b) Top views from cytoplasmic and periplasmic side. (c,d) Side views as seen from the fourfold symmetry axis of the tetramer (c) and from outside the tetramer (d). The helices of GIpF are highly tilted ( $\mathrm{H} 1: 28^{\circ}$ against $\mathrm{z}-\mathrm{direction}, \mathrm{H} 2: 23^{\circ}, \mathrm{H} 3: 37^{\circ}, \mathrm{H} 4$ : $22^{\circ}, \mathrm{H} 5: 21^{\circ}, \mathrm{H} 6: 38^{\circ}$ ). The maps are generated using the programs DI NO (Visualizing Structural Biology 2000, http://www.bioz.unibas.ch/ $\sim$ xray/dino) and POVRAY (http://www.povray.org). (e) Topology model of GIpF , showing six transmembrane helices and two short helices in the functional loops B and E. The N-terminal 10-His tag and 11 spacer amino acids are shown in gray, while stars mark the NPA motifs. Black circular disks indicate the sites proposed to face the channel, based on the atomic model (Murata et al., 2000) and alignment of 164 sequences (Heymann and Engel, 2000). GIpF has in comparison with AQP1 inserts of 18 residues in the periplasmic loop C and 13 residues in loop $\mathrm{E}$, as displayed in red. The proposed interpretation of the peripheral densities D1 through D5 is marked by yellow areas. 
a

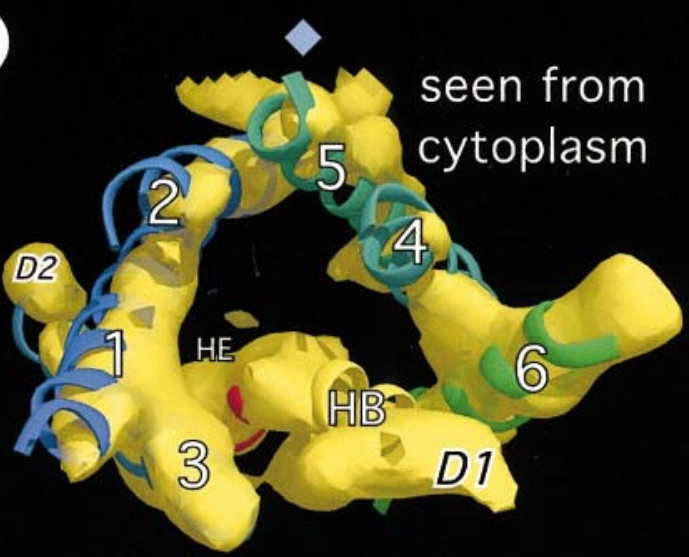

C

Cytoplasm (b)

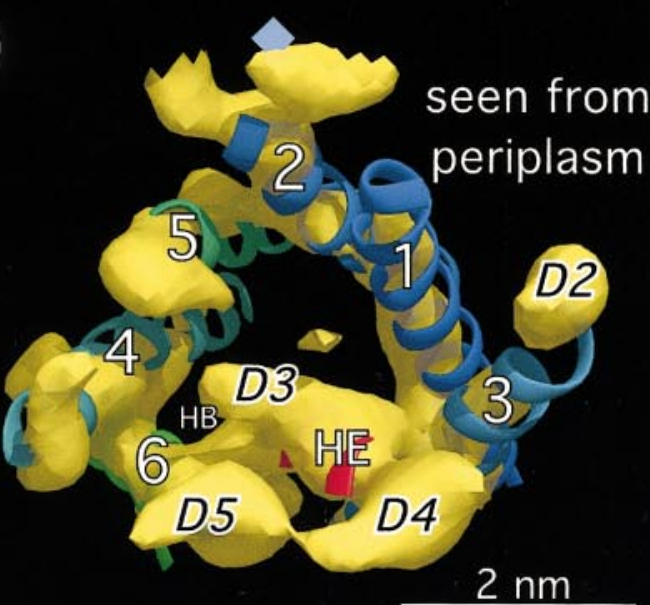

d)

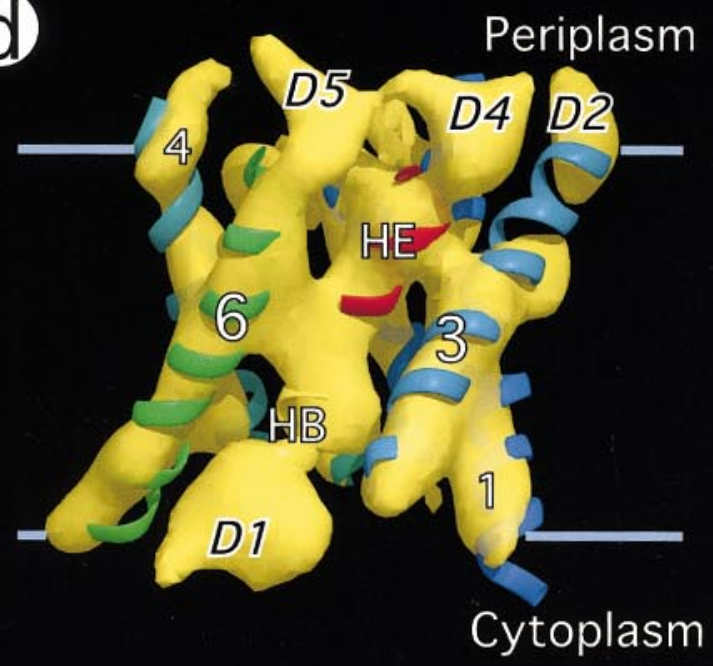

(e)

(A) 1$\left.)^{\circ}\right)(8)$

๑०0ब (ब)

BQ(1) B(1)

(1) 150 (1)

(ब) 6 (1) $(101)(1)$ (A)

(A) $(A)$ (A)

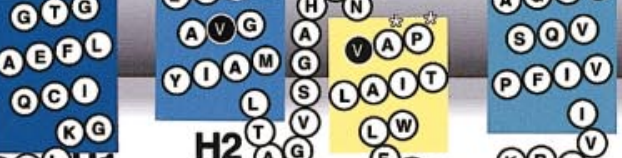

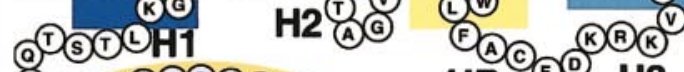

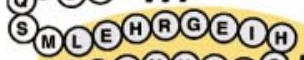
(A) $(A)$ A $A$ S S S $D 1$

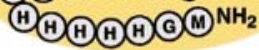

(C)(A) (A) (A)

$H B$

() $\mathrm{H3}$
D2 D3

5요

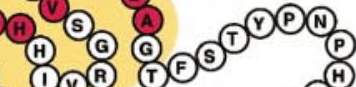

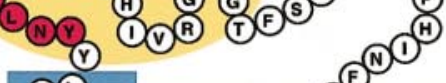
(N) (1) (1) के (A) HOOC (L)SA) ROEESOCH (क) (T)

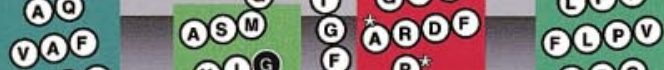
(1) $A$ (1) (D) 10 (1) (1) 9 (1) $(10)$ (1) 100 (A) (1) (1) (1) H4 (ब)(6)

Periplasm . (2) HE H6 ${ }^{\circledR}$ OPD(DABAC Cytoplasm 
ness and phase origin were determined, the images were merged, imposing p4 symmetry.

The azimuthal projection of the collected threedimensional data set is shown in Fig. 2a. To calculate the 3D map, lattice lines were fitted and interpolated (Fig. 2b). The phase residuals of the merged data indicated significant structure information up to $6.9-\AA$ (Table I). Because spots from the two overlapping lattices were not sufficiently separated in the electron diffraction patterns recorded with our IK CCD, amplitude data obtained from the images were taken to calculate the 3D density map.

\section{The 6.9-^ 3D Map of GlpF}

The emerging 3D density map (Fig. 3) reveals two tetramers in opposite orientations (correlation coefficient 0.69 after aligning one tetramer with the inverted second tetramer, but 0.50 without inversion). The tetramers exhibit an overall similarity to the AQP1 tetramer (Walz et al., 1997), but show on one side a density peak about the fourfold axis. The GIpF monomer (Fig. 4) comprises six highly tilted rod-like structures that surround a central density. Since the two tetramers of one unit cell are not related by crystallographic symmetry (Braun et al., 2000), the reproducibility of pertinent features can be assessed by superposition of the two independent monomers (Figs. 4a and 4b; shaded and wire frame surfaces). The resolution estimated from their Fourier shell correlation coefficient is $8.1 \AA$ (van Heel, 1987), compatible with the resolution limits imposed for merging the data (Table I and Fig. 2a). Compared to the AQP1 monomer rendered at 6.9- $\AA$ resolution (Fig. 4C), GlpF exhibits distinct additional domains (D1-D5) that are present in both independent 3D maps, although D3 is weaker in the shaded map (Figs. 4a and 4b). Therefore, these domains are genuine features of the GlpF structure that are probably related to sequence differences between GIpF and AQP1.

\section{Comparison of GIpF with AQP1}

To compare the 6.9- $\AA$ structure of GIpF with the atomic model of AQP1 (Murata et al., 2000), the independent GIpF monomers shown in Fig. 4 were merged and their sidedness was determined by three criteria: (1) The side protruding further out of the membrane was identified as extracellular as observed for AQP0, AQP1, and AqpZ by atomic force microscopy (Engel et al., 2000). (2) A systematic search for the best-fitting orientations of an $\alpha$-carbon model of the structure of AQP1 in the 3D map of GlpF (de Groot et al., 2000) showed that the orientation with the more protruding surface being extracellular fits better (with a "rottrans" score of 0.62; see Materials and Methods) than orientations with the opposite sidedness (with a score of 0.51). (3) The deviation of helix 1 from the quasi-twofold symme try of the monomer with respect to an axis in the membrane plane (Cheng et al., 1997; Li et al., 1997), which is more pronounced in GIpF than in AQP1, allowed helix 1 and hence the sidedness to be identified. This assignment corroborated the results of criteria (1) and (2).

The superposition of the GIpF monomer and the AQP1 atomic model shown as ribbons (Fig. 5) confirms the strong similarity of these molecules as al ready suggested by Fig. 4 . While the match of the two structures is pronounced in their centers, distinct differences are seen at the cytoplasmic surface (D1) and the extracellular surface (D2-D5). Considering the GIpF sequence (Fig. 5e), additional residues at the $\mathrm{N}$-terminus are tentatively assigned to domain $\mathrm{D1}$, the additional residues in loops $\mathrm{C}$ (Y109-H119 and V126-A132) are assigned to the extension of helix 3 (D2) and a density closer to the interior of the monomer (D3), and additional residues in loop E (A217-D229) are assigned to the extensions of helix HE (D4) and helix 6 (D5). Since loop regions are always less well defined than the helices, higher resolution data are required to prove this hypothesis. However, the 6.9- $\AA$ map clearly shows that the additional residues in loops $\mathrm{C}$ and $\mathrm{E}$ do not restrict the channel but are found at the surface of the protein, located mainly at the periphery of the tetramer.

\section{Homology Modeling of GlpF Channel}

The pronounced structural similarity of GlpF and AQP1 shown in Figs. 5a-5d and the strong conservation of critical residues (Fig. 5e; Heymann and Engel, 2000) prompted us to build a model of the glycerol channel based on the AQP1 atomic model: In a first step all AQP1 residues were replaced by residues of GlpF , using the alignment based on multiple sequence analysis shown in Fig. 5e. This re sulted in an overall reduction of the mass protruding into the channel by approximately $100 \mathrm{Da}$. With the exception of the site with the two aromatic residues W42 and Y138, a general widening of the channel was observed. A subsequent database search for possible orientations of Trp and Tyr in environments with similar backbone conformations revealed an alternative and equally probable orientation of these residues that eliminated the narrowing of the channel at this position (Vriend, 1990). Projection maps of both the atomic model of AQP1 and the putative $\mathrm{GlpF}$ model were then calculated to compare them with the experimental projection maps. While the excellent correlation between the calculated and experimental map of AQP1 validates the approach to 




FIG. 6. Atomic modeling of the GIpF based on the structure and sequence homology of GIpF and AQP1 and the atomic structure of AQP1 (Murata et al., 2000). (a) The 3.5- $\AA$ AQP1 projection structure with the 3D map of the monomer at 6.9- $\AA$ resolution (blue) and the projection of the atomic model (brown). (b) The 3.7- $\AA$ GlpF projection structure with the 3D map of the monomer at $6.9-\AA$ resolution (yellow) and the projection of the constructed model of GlpF. The dotted line indicates the pseudo-twofold symmetry axis of the monomer. (c,d) Channels of AQP1 (c) and GIpF (d), calculated by HOLE (Vriend, 1990). The F 24 marked in red correlates with the bend in the AQP1 channel. (e) The amphiphilic nature of the GIpF channel is revealed by color coding the accessible surfaces: green, aromatic residues; yellow, hydrophobic residues; white, polar residues; blue, positively charged residues.

test an atomic model with a high-resolution projection map (Fig. 6a), the wider pore region observed in the experimental projection map of GIpF is also observed in the calculated map (Fig. 6b). This suggests that the structural model of the glycerol channel is correct within the experimental error.

To reveal the shape of the channel, the accessible channel surfaces were probed with the program HOLE (Smart et al., 1996). While AQP1 exhibits a distinct eyelet with a minimal diameter of $2.8 \AA$ that is close to a pronounced bend of the channel (Fig. 6c), the GIpF channel appears to be slightly larger, rather uniform in size and free of bends, having a minimal pore diameter of $3.3 \AA$ (Fig. 6d). The bend in the AQP1 channel is related to F 24, which is replaced by a Leu in GIpF. While $63 \%$ of the so far identified water channels have the residue pairs Phe/X (with X being mostly Leu) that form part of the eyelet (Murata et al., 2000), 15 of 19 GLP subtypes exhibit the pair Leu/Leu, 2 exhibit Leu/M et, and 2 exhibit Leu/Phe (Heymann and Engel, 2000). In some aquaporin subtypes, a Tyr is present instead of F24. Among these are AQP0, reported to possess $\mathrm{pH}$-dependent water capacity (Nemeth-Cahalan and Hall, 2000; Zeuthen and Klaerke, 1999), and AQP6, which becomes a chloride channel at pH 5.5 (Yasui et al., 1999). This indicates a possible role of $Y 24$ in channel gating. Finally, the channel-surrounding residues exhibit an amphiphilic nature: One side 
is hydrophobic, while the other is hydrophilic (Fig. $6 e)$. Thus, the glycerol channel has properties akin to maltoporin with its greasy slide that promotes passage of maltodextrins (Schirmer et al., 1995). This property may partially explain the contradiction of glycerol diffusion but limited water capacity (Maurel et al., 1994).

\section{CONCLUSIONS}

Electron crystallography combined with homology modeling is a powerful approach to elucidate the structure of a membrane protein. Strong sequence conservation within the aquaporin family, the structural homology between GIpF and AQP1, and the availability of an atomic model of AQP1 provided a solid basis for modeling of the GIpF channel.

Note added in proof. The validity of this approach can now be assessed by comparing the model with the structure determined by X-ray crystallography to $2.2 \AA$ (Fu et al., 2000), which has been published after submission of the work presented here.

We thank Deryck Mills for help with the J eol 3000 SFF microscope, Richard Henderson and Vinzenz Unger for continuous advice and support concerning image treatment, and Helmut Grubmüller for his help in modeling the GIpF. We are indebted to Yoshinori Fujiyoshi, Kaoru Mitsuoka, and Kazuyoshi Murata for providing unpublished data, and we thank them as well as Simon Scheuring for fruitful discussions. The work was supported by the Swiss National Foundation for Scientific Research (NF Grant 4036-44062 to A.E.), the M. E. Müller Foundation of Switzerland, the National Institutes of Health (to P.A.), and the EU Biotech program (Grant BIO4-CT98-0024 to H.G.).

\section{REFERENCES}

Braun, T., Philippsen, A., Wirtz, S., Borgnia, M. J ., Agre, P., Kühlbrandt, W., Engel, A., and Stahl berg, H. (2000) The $3.7 \AA$ projection map of the glycerol facilitator GIpF: A variant of the aquaporin tetramer, EMBO Rep. 2, 183-189.

Bron, P., Lagrée, V., Froger, A., Rolland, R., Hubert, J . F., Delamarche, C., Deschamps, S., Pellerin, I., Thomas, D., and Haase, W. (1999) Oligomerization state of MIP proteins expressed in Xenopus oocytes as revealed by freeze-fracture electron-microscopy analysis, J. Struct. Biol. 128, 287-296.

Cheng, A., van Hoek, A. N., Yeager, M., Verkman, A. S., and Mitra, A. K. (1997) Three-dimensional organization of a human water channel, Nature 387, 627-630.

Collaborative Computational Project, N. (1994) The CCP4 Suite: Programs for protein crystallography, Acta Crystallogr. 50, 760-763.

Crowther, R. A., Henderson, R., and Smith, J. M. (1996) MRC image processing programs, J . Struct. Biol. 116, 9-16.

de Groot, B. L., Heymann, J . B., Engel, A., Mitsuoka, K., Fujiyoshi, Y., and Grubmüller, H. (2000) The fold of human aquaporin 1, J. Mol. Biol. 300, 987-994.

Dubochet, J ., Adrian, M., Chang, J.-J ., Homo, J .-C., Lepault, J ., McDowall, A. W., and Schultz, P. (1988) Cryo-electron microscopy of vitrified specimens, Quart. Rev. Biophys. 21, 129-228.

Engel, A., Fujiyoshi, Y., and Agre, P. (2000) The importance of aquaporin water channel protein structures, EMBO J . 19, 800806.
Fotiadis, D., Hasler, L., Müller, D. J ., Stahlberg, H., Kistler, J ., and Engel, A. (2000) Surface tongue-and-groove contours on lens MIP facilitate cell-to-cell adherence, J. Mol. Biol. 300, 779-789.

Froger, A., Tallur, B., Thomas, D., and Delamarche, C. (1998) Prediction of functional residues in water channels and related proteins, Protein Sci. 7, 1458-1468.

Fu, D., Lisbon, A., Miercke, L. J . W., Weitzman, C., Nollert, P., Krucinski, J ., and Stroud, R. M. (2000) Structure of a glycerolconducting channel and the basis for its selectivity, Science 290, 481- 486.

Gorin, M. B., Yancey, S. B., Cline, J ., Revel, J .-P., and Horwitz, J . (1984) The major intrinsic protein (MIP) of the bovine lens fiber membrane: Characterization and structure based on CDNA cloning, Cell 39, 49-59.

Hasler, L., Walz, T., Tittmann, P., Gross, H., Kistler, J ., and Engel, A. (1998) Purified lens major intrinsic protein (MIP) forms highly ordered tetragonal two-dimensional arrays by reconstitution, J. Mol. Biol. 279, 855- 864.

Henderson, R., Baldwin, J. M., Ceska, T. A., Zemlin, F., Beckmann, E., and Downing, K. H. (1990) Model for the structure of bacteriorhodopsin based on high-resolution electron cryomicroscopy, J. Mol. Biol. 213, 899-929.

Henderson, R., Baldwin, J . M., Downing, K. H., Lepault, J ., and Zemlin, F . (1986) Structure of purple membrane from Hal obacterium halobium: Recording, measurement and evaluation of electron micrographs at $3.5 \AA$ resolution, Ultramicroscopy $\mathbf{1 9}$, 147-178.

Heymann, B., and Engel, A. (2000) Structural clues in the sequences of the aquaporins, J. Mol. Biol. 295, 1039-1053.

Heymann, J. B., and Engel, A. (1999) Aquaporins: Phylogeny, structure and physiology of water channels, News Physiol. Sci. 14, 187-193.

Hirai, T., Murata, K., Kimura, Y., and Fujiyoshi, Y. (1999) Trehalose embedding technique for high-resolution electron crystallography: Application to structural study on bacteriorhodopsin, J . Electron Microsc. 48, 653- 685.

J ung, J. S., Preston, G. M., Smith, B. L., Guggino, W. B., and Agre, P. (1994) Molecular structure of the water channel through aquaporin CHIP. The hourglass model, J . Biol. Chem. 269, 14648-14654.

Kunji, E. R., von Gronau, S., Oesterhelt, D., and Henderson, R. (2000) The three-dimensional structure of halorhodopsin to $5 \AA$ by electron crystallography: A new unbending procedure for two-dimensional crystals by using a global reference structure, Proc. Natl. Acad. Sci. USA 97, 4637- 4642.

Lagree, V., Froger, A., Deschamps, S., Hubert, J . F., Delamarche, C., Bonnec, G., Thomas, D., Gouranton, J., and Pellerin, I. (1999) Switch from an aquaporin to a glycerol channel by two amino acids substitution, J. Biol. Chem. 274, 6817- 6819.

Lagree, V., Froger, A., Deschamps, S., Pellerin, I., Delamarche, C., Bonnec, G., Gouranton, J., Thomas, D., and Hubert, J . F. (1998) Oligomerization state of water channels and glycerol facilitators. Involvement of Ioop E, J . Biol. Chem. 273, 3394933953.

Li, H., Lee, S., and J ap, B. K. (1997) Molecular design of aquaporin-1 water channel as revealed by electron crystallography, Nat. Struct. Biol. 4, 263-265.

Maurel, C., Reizer, J ., Schroeder, J . I., Chrispeels, M. J ., and Saier, M. H., J r. (1994) Functional characterization of the Escherichia coli glycerol facilitator, GlpF, in Xenopus oocytes, J . Biol. Chem. 269, 11869-11872.

Mitsuoka, K., Murata, K., Walz, T., Hirai, T., Agre, P., Heymann, J. B., Engel, A., and Fujiyoshi, Y. (1999) The structure of 
aquaporin-1 at $4.5-\AA$ resolution reveals short alpha-helices in the center of the monomer, J . Struct. Biol. 128, 34-43.

Murata, K., Mitsuoka, K., Hirai, T., Walz, T., Agre, P., Heymann, J . B., Engel, A., and Fujiyoshi, Y. (2000) Structural determinants of water permeation through aquaporin-1. Nature 407, 599- 605.

Nemeth-Cahalan, K. L., and Hall, J. E. (2000) pH and calcium regulate the water permeability of aquaporin 0, J. Biol. Chem. 275, 6777- 6782.

Park, J . H., and Saier, M. H., J r. (1996) Phylogenetic characterization of the MIP family of transmembrane channel proteins, J. Membr. Biol. 153, 171-180.

Preston, G. M., and Agre, P. (1991) I solation of the cDNA for erythrocyte integral membrane protein of 28 kilodaltons: Member of an ancient channel family, Proc. Natl. Acad. Sci. USA 88, 11110-11114.

Preston, G. M., Carroll, T. P., Guggino, W. B., and Agre, P. (1992) Appearance of water channels in Xenopus oocytes expressing red cell CHIP28 protein, Science 256, 385-387.

Ringler, P., Borgnia, M. J ., Stahlberg, H., Maloney, P. C., Agre, P., and Engel, A. (1999) Structure of the water channel AqpZ from Escherichia coli revealed by electron crystallography, J . Mol. Biol. 291, 1181-1190.

Scheuring, S., Ringler, P., Borgnia, M., Stahlberg, H., Muller, D. J., Agre, P., and Engel, A. (1999) High resolution AFM topographs of the Escherichia coli water channel aquaporin $Z$, EMBO J . 18, 4981- 4987.

Scheuring, S., Tittmann, P., Stahlberg, H., Ringler, P., Borgnia, M., Agre, P., Gross, H., and Engel, A. (2000) The aquaporin sidedness revisited, J. Mol. Biol. 299, 1271-1278.
Schirmer, T., Keller, T. A., Wang, Y. F., and Rosenbusch, J . P. (1995) Structural basis for sugar translocation through maltoporin channels at $3.1 \AA$ resolution, Science 267, 512514.

Smart, O. S., Neduvelil, J. G., Wang, X., Wallace, B. A., and Sansom, M. S. P. (1996) HOLE: A program for the analysis of the pore dimensions of ion channel structural models, J. Mol. Graphics 14, 354-360.

van Heel, M. (1987) Similarity measures between images, Ultramicroscopy 21, 95-100.

Voegele, R. T., Sweet, G. D., and Boos, W. (1993) Glycerol kinase of Escherichia coli is activated by interaction with the glycerol facilitator, J . Bacteriol. 175, 1087-1094.

Vriend, G. (1990) WHAT IF: A molecular modeling and drug design program, J. Mol. Graphics 8, 52-56, 29.

Walz, T., Hirai, T., Murata, K., Heymann, J. B., Mitsuoka, K., Fujiyoshi, Y., Smith, B. L., Agre, P., and Engel, A. (1997) The three-dimensional structure of aquaporin-1, Nature 387, 624627.

Walz, T., Smith, B. L., Agre, P., and Engel, A. (1994) The threedimensional structure of human erythrocyte aquaporin CHIP, EMBO J . 13, 2985-2993.

Yasui, M., Hazama, A., Kwon, T. H., Nielsen, S., Guggino, W. B., and Agre, P. (1999) Rapid gating and anion permeability of an intracellular aquaporin, Nature 402, 184-187.

Zeuthen, T., and Klaerke, D. A. (1999) Transport of water and glycerol in aquaporin 3 is gated by $\mathrm{H}(+), \mathrm{J}$. Biol. Chem. 274, 21631-21636. 